\title{
Comparing Combinatory Reduction Systems and Higher-order Rewrite Systems
}

\author{
Vincent van Oostrom ${ }^{1}$ and Femke van Raamsdonk ${ }^{2}$ \\ 1 Department of Mathematics and Computer Science, Vrije Universiteit, De \\ Boelelaan 1081a, $1081 \mathrm{HV}$ Amsterdam, The Netherlands, email:

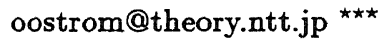 \\ 2 CWI, P.O. Box 94079, 1009 GB Amsterdam, The Netherlands, email: femke@cwi.nl
}

\begin{abstract}
In this paper two formats of higher-order rewriting are compared: Combinatory Reduction Systems introduced by Klop and Higherorder Rewrite Systems defined by Nipkow. Although it always has been obvious that both formats are closely related to each other, up to now the exact relationship between them has not been clear. This was an unsatisfying situation since it meant that proofs for much related frameworks were given twice. We present two translations, one from Combinatory Reduction Systems into Higher-Order Rewrite Systems and one vice versa, based on a detailed comparison of both formats. Since the translations are very 'neat' in the sense that the rewrite relation is preserved and (almost) reflected, we can conclude that as far as rewrite theory is concerned, Combinatory Reduction Systems and Higher-Order Rewrite Systems are equivalent, the only difference being that Combinatory Reduction Systems employ a more 'lazy' evaluation strategy. Moreover, due to this result it is the case that some syntactic properties derived for the one class also hold for the other.
\end{abstract}

The research of the second author is supported by NWO/SION project 612-316-606.

\section{Introduction}

This paper is concerned with a comparison of two formats of higher-order rewriting: Combinatory Reduction Systems (CRSs) as introduced by Klop [Klo80] and Higher-order Rewrite Systems (HRSs) as introduced by Nipkow [Nipa].

Inspired by Aczel [Acz78], Klop defined CRSs in [Klo80] as first-order term rewriting systems possibly with bound variables, so as to include both first-order rewrite systems such as Curry's Combinatory Logic and rewrite systems with bound variables such as Church's $\lambda$-calculus. The point was that a large amount of syntactic rewrite theory could be developed for this framework.

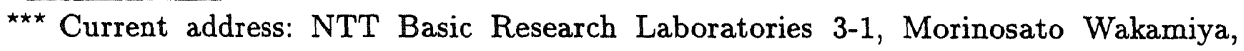
Atsugi-Shi, Kanagawa Pref., 243-01, Japan 
In [Nipa], Nipkow introduces HRSs as a generalisation of first-order rewrite systems to terms with higher-order functions and bound variables. Furthermore, HRSs were designed to have the same logical basis as systems like Isabelle [Pau90] and $\lambda$ Prolog [NM88]. That is, a typed $\lambda$-calculus is used as a meta-language.

These different objectives have led to surprisingly large differences in the presentation of these systems. For CRSs the meta-language, i.e. the language in which the notions of term, substitution and rewrite step are expressed, is left implicit in the presentation. For HRSs the meta-language is Church's $\lambda \rightarrow$-calculus of simply typed $\lambda$-terms with $\beta$ as rewrite rule. In the case of CRSs, the introduction of a special purpose meta-language makes the definition quite involved. However, a closer inspection shows that in fact the meta-language of CRSs is (a polyadic version of) $\lambda$-calculus with developments (or let-expressions), denoted by $\underline{\lambda}$. See $[\mathrm{Klo80}$, Sec. I.3.5] or [Bar84, $\S 11.1 .3]$ for details.

Once we have made the meta-language of CRSs explicit, we can compare both formats by comparing their respective meta-languages. Comparing is done by giving encodings of one system into the other and vice versa. The encoding of CRSs into HRSs is straightforward because $\underline{\lambda}$-calculus can be encoded into $\lambda \rightarrow$-calculus. The encoding of HRSs into CRSs is somewhat more involved; $\lambda \overrightarrow{\text { - }}$ calculus cannot be encoded directly into $\underline{\lambda}$-calculus. For example, the latter does enjoy the disjointness property (rewriting preserves disjointness, cf. [Klo80, pg. $38]$ ), while the former doesn't. In general, in $\lambda^{\rightarrow}$-calculus rewrite sequences can be longer than in $\underline{\lambda}$-calculus. Our solution is to add an explicit $\beta$-rule (and a symbol for application) to the encoding of an HRS. A rewrite step in the HRS is then simulated by a rewrite step in the CRS possibly followed by an explicit $\beta$-reduction to normal form. More precisely, let $\mathcal{C}$ be a CRS and $\mathcal{H}$ be a HRS. We write $\rightarrow_{\mathcal{C}}$ and $\rightarrow_{\mathcal{H}}$ for their rewrite relations. Translating is denoted by $\langle-\rangle$, and reduction to normal form with respect to the explicit $\beta$-rule is written as $\rightarrow !$. Then we have

$$
\begin{aligned}
\langle\rightarrow \mathcal{C}\rangle & =\rightarrow\langle\mathcal{C}\rangle \\
\langle\rightarrow \mathcal{H}\rangle & =\rightarrow\langle\mathcal{H}\rangle \cdot \underset{\beta}{\stackrel{!}{\vec{\beta}}}
\end{aligned}
$$

if the relations are restricted to the set of translated terms.

The naturality of an an encoding can be measured by the properties it preserves and reflects. Our encoding of CRSs into HRSs both preserves and reflects the main property of rewrite systems, i.e. whether one term rewrites (in one step) to another. This allows for a confluence proof for orthogonal CRSs via a proof of confluence for orthogonal HRSs. As noted above the translation the other way around is not that nice. The HRS is simulated by a more refined CRS; 'giant' HRS-steps are simulated by many 'small' CRS-steps. This is analogous to the way in which $\lambda$-calculus is simulated by the $\lambda \sigma$-calculus defined in [ACCL90]. Of course, not every step in the refined system is reflected in the original HRS, but still we can say something: every rewrite sequence between encodings of HRS-terms is reflected in the original HRS. Again, this allows for a confluence 
proof for orthogonal HRSs via a proof of confluence for orthogonal CRSs. For the moment being, we have only considered use of our translation for confluence results.

Our comparison only considers CRSs versus HRSs. There are some more alternatives for higher-order rewriting, such as Khasidashvili's Expression Reduction Systems [Kha90] and Takahashi's Conditional Lambda Calculi [Tak]. We claim that the main differences between these and CRSs (or HRSs) are of a syntactic nature.

The paper is organised as follows. In section 2 we will discuss in detail the difference between CRSs and HRSs by first considering only terms, and next also the rewrite relation on terms. In section 3 we define a translation from CRSs into HRSs and, using this translation, we give a confluence proof for orthogonal CRSs. The translation from HRSs into CRSs is presented in section 4, again the translation is used to give a confluence proof, now for orthogonal HRSs. Section 5 concludes the paper with some discussion on higher-order rewriting. The reader is assumed to be familiar with term rewriting and (simply typed) $\lambda$-calculus. For the induction proofs we refer the reader to CWI Report CS-R9361 or VU Report IR-333 [OR93], both with the same title as this paper.

NOTATION. We adhere mostly to the notations introduced by Klop for CRSs, and Nipkow for HRSs. Since their introduction both formats have been subject to some change and we will use their most recent presentations, viz. [KOR93] for CRSs and [Nipb] for HRSs. The most notable change is the use of the functional format for CRSs instead of the applicative one of [Klo80]. The reason for choosing the functional format is that it is closer to the usual notation for term rewriting systems. Moreover, in applicative CRSs the object-language application symbol is left implicit in the notation, while for HRSs the meta-language application synbol is left implicit, which would possibly give rise to confusion in comparing these formats.

\section{Comparing the Syntax}

We first restrict attention to term formation, since already on that level some important differences between CRSs and HRSs are manifest. Next, we consider rule formation and finally the generation of the rewrite relation.

\subsection{Term Formation}

CRS Terms. A CRS $\mathcal{C}$ is a pair $(\mathcal{A}, \mathcal{R})$, where $\mathcal{A}$ is its alphabet and $\mathcal{R}$ its set rewrite or reduction rules. (Because of the termination connotation of the word 'reduction' we will use it only in the case of normalising rewrites.) In a CRS a distinction is made between metaterms and terms. The left- and right-hand side of a rule are metaterms, but the rewrite relation is a relation on terms.

The alphabet $\mathcal{A}$ of a $\mathrm{CRS}(\mathcal{A}, \mathcal{R})$ consists of 
- symbols for variables $x y z \ldots$,

- a symbol [-]- for the abstraction operator,

- symbols for operators with a fixed arity $F G H$...,

- symbols for metavariables with a fixed arity $Z Z_{0} Z_{1} \ldots$

The set MTerms of metaterms is the least set such that

(1) $x \in$ MTerms for every variable $x$,

(2) $[x] t \in$ MTerms for a variable $x$ and $t \in$ MTerms,

(3) $F\left(t_{1}, \ldots, t_{n}\right) \in$ MTerms if $t_{1}, \ldots, t_{n} \in M$ Terms and $F$ is an $n$-ary operator,

(4) $Z\left(t_{1}, \ldots, t_{n}\right) \in$ MTerms if $t_{1}, \ldots, t_{n} \in$ MTerms and $Z$ is an $n$-ary metavariable.

The set Terms of terms consists of all metaterms without metavariables. In a term or metaterm of the form $[x] t$, we call $t$ the scope of $[x]$. A variable $x$ occurs free in a term or metaterm if it is not in the scope of an occurrence of $[x]$. A variable $x$ occurs bound otherwise. A term is called closed if all variables occur bound. Only variables (and no metavariables) can be bound by the abstraction operator. We will sometimes write $\left[x_{1} \ldots x_{n}\right] t$ for $\left[x_{1}\right] \ldots\left[x_{n}\right] t$.

Let $\square$ be a fresh symbol. A context is a term with one or more occurrences of $\square$. A context with exactly one occurrence of $\square$ is written as $C[]$, and one with $n$ occurrences of $\square$ as $C[, \ldots$,$] . If C[, \ldots$,$] is a context with n$ occurrences of $\square$ and $t_{1}, \ldots, t_{n}$ are terms, then $C\left[t_{1}, \ldots, t_{n}\right]$ denotes the result of replacing from left to right the occurrences of $\square$ by $t_{1}, \ldots, t_{n}$.

Example 1. The alphabet of $\lambda$-calculus in CRS format contains two symbols for operators: a unary operator $\lambda$ for $\lambda$-abstraction and a binary operator @ for application. Examples of some $\lambda$-terms written in CRS notation:

$$
\begin{aligned}
& \lambda([x] x) \text { for } \lambda x \cdot x, \\
& @(x, y) \text { for } x y, \\
& \lambda([x] @(y, x)) \text { for } \lambda x . y x, \text { and } \\
& @(\lambda([x] x), y) \text { for }(\lambda x . x) y .
\end{aligned}
$$

Because of the very liberal term formation in the CRS framework, many terms can be formed from the alphabet consisting of $\lambda$ and @ that do not correspond to any $\lambda$-term. These kind of terms are called 'junk'. In general, it is often necessary to consider a CRS with a restricted set of terms, that has to be closed under rewriting. If one wants to stress the point that only a subset of the set of terms is considered, one speaks about sub-CRSs.

HRS Terms. A HRS $\mathcal{H}$ is a pair $(\mathcal{A}, \mathcal{R})$ whith $\mathcal{A}$ an alphabet and $\mathcal{R}$ a set of rules. Term formation is specified using $\lambda \rightarrow$-calculus, Church's simply typed $\lambda$-calculus. (Simple) types are formed from base types and the function type constructor $\rightarrow$. Types are denoted by $\sigma, \tau, \ldots$

The alphabet $\mathcal{A}$ of a HRS $\mathcal{H}=(\mathcal{A}, \mathcal{R})$ consists of

- symbols for typed variables $x y z \ldots$, 
- a distinguished symbol $\lambda$ for abstraction,

- symbols for typed operators $F G H \ldots$..

Typed terms are formed from abstraction and application, which is written by juxtaposition, according to the following rules:

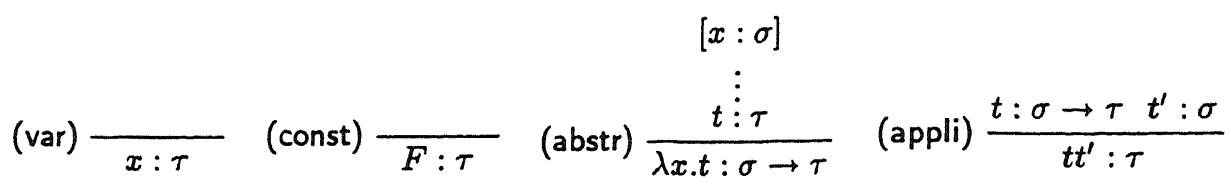

Although environments are not made explicit, we take it for granted that variables and constants cannot have more than one type. So every typable term has a unique type. Like in $\lambda$-calculus, a variable $x$ occurs bound in a term if it occurs in the scope of a $\lambda x$, and it occurs free otherwise.

Let $\square$ be a fresh symbol of some base type. A context is a term with one or more occurrences of $\square$. Like in the CRS case, a context with exactly one occurrence of $\square$ is written as $C[]$, and one with $n$ occurrences of $\square$ as $C[, \ldots$,$] .$ If $C[, \ldots$,$] is a context C[, \ldots$,$] with n$ occurrences of $\square$ and $t_{1}, \ldots, t_{n}$ are terms of appropriate base type, then $C\left[t_{1}, \ldots, t_{n}\right]$ denotes the result of replacing from left to right the occurrences of $\square$ by $t_{1}, \ldots, t_{n}$.

Only terms (and contexts) in long $\eta$-normal form will be considered.

Definition 1. The long $\eta$-normal form of a $\lambda \rightarrow$-term $s$ is obtained as follows. Replace repeatedly subterms $t$ of $s$ by $\lambda x$.tx provided $x$ doesn't occur free in $t, t$ is not of the form $\lambda y . t_{0}$ and $t$ doesn't occur in a subterm of the form $t u$. This has the effect that all subterms are provided with the right number of arguments.

Example 2. In the representation of untyped $\lambda$-calculus as a HRS, we have only one base type 0 . The alphabet contains two symbols for operators, namely app : $0 \rightarrow(0 \rightarrow 0)$ for application and abs : $(0 \rightarrow 0) \rightarrow 0$ for $\lambda$-abstraction. Some examples of $\lambda$-terms in this notation are:

$$
\begin{aligned}
& \text { abs }(\lambda x . x) \text { for } \lambda x . x, \\
& \text { app } x y \text { for } x y, \\
& \text { abs }(\lambda x . \text { app } y x) \text { for } \lambda x . y x, \\
& \text { app }(\operatorname{abs}(\lambda x . x)) y \text { for }(\lambda x . x) y \text {. }
\end{aligned}
$$

Like in the CRS case, the HRS-representation of $\lambda$-calculus contains junk. For instance, the term $\lambda x . x$ doesn't correspond to any $\lambda$-term. Note that all $\lambda$ terms and the variables occurring in them have type 0 in this notation. This example illustrates that a notion of sub-HRS, analogous to the notion of subCRS, is called for. Furthermore, the example shows that properties, such as strong normalisation, of the meta-language $(\lambda \rightarrow$-calculus $)$ have no bearing on properties of the object language ( $\lambda$-calculus). 
Comparing Term Formation. We discuss the two most important differences between both formats.

In CRSs metaterm formation is given by a direct inductive definition. Function symbols and metavariables come equipped with an arity and metaterms are formed by supplying these symbols with the right number of arguments. Terms are metaterms not containing metavariables.

In HRSs a direct inductive definition of terms is circumvented by making use of $\lambda \rightarrow$-calculus term formation. Function symbols come as constants equipped with a type and are combined using the formation rules of $\lambda \rightarrow$-calculus. Attention is then restricted to terms in long $\eta$-normal form. Most of the time, except at intermediate stages of a computation, attention is further restricted to terms in long $\beta \eta$-normal form.

Note that the typing does not mean that only typed systems can be written as HRSs; the typing takes place on metalevel. If an untyped system is represented as a HRS, then only one base type 0 is used and all terms of the HRS corresponding to a term in the untyped system we are considering, are of type 0 . The base type 0 can be thought of as the set of all well-formed terms. The statement $t: 0 \mathrm{can}$ be read as ' $t$ is a well-formed term'.

Typing in this way, such that well-formed terms are of base type, actually establishes two things. For discussing them, first the arity and the order of a type are defined.

Definition 2. The arity $\operatorname{Ar}(\sigma)$ of a type $\sigma$ is inductively defined as follows:

$$
\begin{aligned}
\operatorname{Ar}(\sigma) & =0(\text { if } \sigma \text { is a base type) } \\
\operatorname{Ar}(\sigma \rightarrow \tau) & =1+\operatorname{Ar}(\tau)
\end{aligned}
$$

The order $\operatorname{Ord}(\sigma)$ of a type $\sigma$ is defined as follows:

$$
\begin{aligned}
\operatorname{Ord}(\sigma) & =0(\text { if } \sigma \text { is a base type }) \\
\operatorname{Ord}(\sigma \rightarrow \tau) & =\max (1+\operatorname{Ord}(\sigma), \operatorname{Ord}(\tau))
\end{aligned}
$$

The arity (order) of a term is defined to be the arity (order) of its type.

First, in a term every operator has exactly as many arguments as prescribed by the arity of its type. This is because terms must be in long $\eta$-normal form. For instance, an operator $F: 0 \rightarrow(0 \rightarrow 0)$ can form a term only if it is provided with two arguments $t_{1}$ and $t_{2}$ of type 0 . So the type of an operator, like the arity of an operator in CRSs, determines how many arguments it should have. Second, in a term all the arguments have the right order, indicating how active they are, or, whether they can be applied to other terms. For example an operator $G:(0 \rightarrow$ $0) \rightarrow 0$ should have one argument of order 1 . The order of an operator cannot be directly expressed in the CRS framework. The arity of an CRS operator only prescribes how many arguments this operator should get, but nothing is specified about the orders these arguments should have. 
The second difference is that in CRSs a distinction is made between metavariables and variables and metaterms and terms. Metavariables occur only in metaterms, which in turn occur only as the left- or right-hand side of rewrite rules. The objects which are rewritten are terms. This distinction is made in order to stress the point that a rewrite rule acts as a scheme, so its left- and right-hand side are not ordinary terms. Taking this point of view, $x$ in $F(x)$-as-a-term is a variable, and $x$ in $F(x)$-as-a-left-or-right-hand-side is a metavariable. In CRS notation, the former is written as $F(x)$ and the latter as $F(Z)$. In HRSs no distinction is made between metavariables and variables, and no distinction is made between ordinary terms on the one hand and left- and right-hand sides of rules on the other hand; they both can be rewritten. The metavariables in CRS-rules correspond to free variables in HRS-rules.

\subsection{Rule Formation}

In this section we will compare the rule formation of CRSs with the one of HRSs. We show that rewrite rules in both formats satisfy equivalent requirements.

CRS Rules. In a CRS, a rewrite rule $l \rightarrow r$ must satisfy the following:

(1) $l$ and $r$ are metaterms,

(2) the head-symbol of $l$ is an operator symbol,

(3) all metavariables in $r$ occur in $l$ as well, $l$ and $r$ are closed,

(4) a metavariable $Z$ in $l$ occurs only in the form $Z\left(x_{1}, \ldots, x_{n}\right)$ with $x_{1}, \ldots, x_{n}$ distinct bound variables.

We call the last condition the pattern-condition.

Example 3. The $\beta$-rule of $\lambda$-calculus, $(\lambda x . M) N \rightarrow M[x:=N]$ is written in CRS format as

$$
@\left(\lambda([x] Z(x)), Z^{\prime}\right) \rightarrow Z\left(Z^{\prime}\right)
$$

The head-symbol of the left-hand side is @, and the metavariables $Z$ and $Z^{\prime}$ occur in both sides.

HRS Rules. A rewrite rule $l \rightarrow r$ in a HRS must meet the following requirements:

(1) $l$ and $r$ are both long $\beta \eta$-normal forms of the same base type,

(2) $l$ is not $\eta$-equivalent to a free variable,

(3) all free variables in $r$ occur free in $l$ as well,

(4) a free variable $z$ in $l$ occurs only in the form $z t_{1} \ldots t_{n}$ with $t_{1}, \ldots, t_{n} \eta$ equivalent to $n$ distinct bound variables.

Like for CRSs, the last condition is called the pattern-condition.

Example 4. The $\beta$-rewrite rule in HRS notation is

$$
\operatorname{app}(\operatorname{abs}(\lambda x . y x)) z \rightarrow y z
$$

with $x, z: 0$ and $y: 0 \rightarrow 0$. 
Comparing Rule Formation. Remembering that metavariables in rewrite rules of CRSs correspond to free variables in rewrite rules, it is not difficult to see that the requirements (1)-(4) of CRS rules correspond to the same ones of HRS rules.

The first condition specifies that rules are built from metaterms for the CRS case. The second one states that left-hand sides must have some structure and the third one that rewriting cannot introduce arbitrary terms. These conditions are familiar from first-order rewriting. The last condition is the pattern-condition. By that condition only names (simple objects), not values (compound objects) can occur as arguments of free variables. Both in the case of CRSs and of HRSs it establishes decidability of unification of patterns, and computability of the rewrite relation, a result of [Mil]. Intuitively this is the case since an instance of a pattern has the same 'global structure' as the pattern itself.

\subsection{Rewrite Step Generation}

Once we know what requirements the rewrite rules should satisfy, we have to define for both formats how rewrite rules are instantiated in order to obtain an actual rewrite step. In both cases, we have to plug in some term in the 'holes' of the rule. In CRSs, the holes in the rule are the metavariables, and in HRSs the free variables. The ways in which metavariables and free variables are assigned a value, are related, but nevertheless essentially different.

For defining substitution for CRSs, a polyadic version of $\underline{\lambda}$-calculus is used. The substitution is performed by replacing a metavariable by a (special form of a) $\lambda$-term, and by reducing, in the term obtained by this replacement, all residuals of $\beta$-redexes that are present in the initial term, i.e. by performing a development (or expanding let-constructs). The well-known result in $\lambda$-calculus that all developments are finite, guarantees that the substitution is well-defined.

For defining substitution for HRSs, like for defining term and rule formation, $\lambda \rightarrow$-calculus is used as a metalanguage. The substitution is performed by replacing a free variable by a term of the same type, and reducing the result of the replacement to $\beta$-normal form. In this case, substitution is well-defined since in $\lambda \rightarrow$-calculus all $\beta$-rewrite sequences eventually terminate.

CRS Rewrite Steps. In order to define assignments for CRSs we first introduce a new concept: the so-called substitutes (cf. [Kah92]). An $n$-ary substitute is an expression of the form $\underline{\lambda}\left(x_{1}, \ldots, x_{n}\right) . s$, where $s$ is a term, $\underline{\lambda}$ a 'metalambda' and $\left(x_{1}, \ldots, x_{n}\right)$ a tuple of $n$ distinct variables, which are considered to be bound by $\underline{\lambda}$ and may be renamed in the usual way. A substitute $\underline{\lambda}\left(x_{1}, \ldots, x_{n}\right) . s$ can be applied to an $n$-tuple of terms $\left(t_{1}, \ldots, t_{n}\right)$, yielding $s$ with $x_{1}, \ldots, x_{n}$ simultaneously replaced by $t_{1}, \ldots, t_{n}$ respectively:

$$
\left(\underline{\lambda}\left(x_{1}, \ldots, x_{n}\right) . s\right)\left(t_{1}, \ldots, t_{n}\right)=s\left[x_{1}:=t_{1} \ldots x_{n}:=t_{n}\right]
$$

An assignment $\sigma$ is a mapping from $n$-ary metavariables to $n$-ary substitutes:

$$
\sigma(Z)=\underline{\lambda}\left(x_{1}, \ldots, x_{n}\right) . s \quad(Z \text { an } n \text {-ary metavariable })
$$


It is extended to a mapping from metaterms to terms in the following way:

$$
\begin{aligned}
x^{\sigma} & =x \\
([x] t)^{\sigma} & =[x] t^{\sigma} \\
\left(F\left(t_{1}, \ldots, t_{n}\right)\right)^{\sigma} & =F\left(t_{1}^{\sigma}, \ldots, t_{n}^{\sigma}\right) \\
\left(Z\left(t_{1}, \ldots t_{n}\right)\right)^{\sigma} & =\sigma(Z)\left(t_{1}^{\sigma}, \ldots, t_{n}^{\sigma}\right)
\end{aligned}
$$

Note that the result of applying $\sigma(Z)$ to $\left(t_{1}^{\sigma}, \ldots, t_{n}^{\sigma}\right)$ in the last clause is indeed a term.

A variable in an instance of a metavariable should be bound only if it is bound in the occurrence of the metavariable. Unintended bindings occur for instance in $(F[x] Z)^{\sigma}$ if $\sigma(Z)=x$, and in $\left(Z\left(Z^{\prime}\right)\right)^{\sigma}$ if $\sigma(Z)=\underline{\lambda}(x) \cdot[y] x$ and $\sigma\left(Z^{\prime}\right)=y$. These problems can be avoided by renaming bound variables. In the following we will assume that this is done whenever necessary.

NOTATION. In this paper we stick to the definition of [KOR93] of substitution as a one-stage process. If we would use $\underline{\lambda}$-calculus as a meta-language, we would obtain substitution as a two-stage process: first replacing the metavariables by the terms assigned to them, and then explicitly developing the $\beta$-redexes. This would yield a presentation closer to the one of HRSs.

Rewrite rules generate a rewrite relation $\rightarrow$ on terms in the following way. If $l \rightarrow r$ is a rewrite rule and $\sigma$ an assignment, then $C\left[l^{\sigma}\right] \rightarrow C\left[r^{\sigma}\right]$ is a rewrite or reduction step, where $C[]$ is some context. A contraction is defined as $l^{\sigma} \rightarrow r^{\sigma}$. The reflexive-transitive closure of $\rightarrow$ is called rewriting and is denoted as $\rightarrow$. If $s \rightarrow t$ then we say that $s$ rewrites to $t$. If we want to make explicit that a rewrite rule $R$ is applied in a rewrite step we write $\rightarrow_{R}$ instead of $\rightarrow$.

HRS Rewrite Steps. In a HRS, an assignment is a finite mapping from variables to terms in long $\beta \eta$-normal form of the same type. Using the variable convention of $\lambda$-calculus, an assignment $\sigma$ is extended to a mapping from terms to terms, in the following way:

$$
\begin{aligned}
F^{\sigma} & =F \quad(\text { for a constant } F) \\
x^{\sigma} & =\sigma(x)(\text { for a variable } x) \\
(\lambda x . t)^{\sigma} & =\lambda x . t^{\sigma} \\
\left(t t^{\prime}\right)^{\sigma} & =t^{\sigma} t^{\prime \sigma}
\end{aligned}
$$

We assume bound variables to be renamed whenever necessary. A rewrite relation $\rightarrow$ on terms in long $\beta \eta$ normal form is generated in the following way. If $l \rightarrow r$ is a rewrite rule and $\sigma$ an assignment, then $C\left[l^{\sigma} \downarrow_{\beta}\right] \rightarrow C\left[r^{\sigma} \downarrow_{\beta}\right]$ is a rewrite step. Here $\downarrow_{\beta}$ denotes $\beta$-reduction to normal form. Such a normal form indeed exists since simply typed $\lambda$-calculus is considered. A contraction is defined as $l^{\sigma} \downarrow_{\beta} \rightarrow r^{\sigma} \downarrow_{\beta}$. The terminology of rewriting is the standard one like in the CRS case. 
Comparing Rewrite Step Generation. In both formats it is the case that the first step in performing a substitution is to replace a 'hole' in the rewrite rule by a kind of ' $\lambda$-term'. Then we compute the result of this replacement. And here the difference lies: since in the case of CRSs we perform only a development of the $\lambda$-terms, there is no reduction of created redexes. On the other hand, to compute the result for HRSs full fledged $\lambda \rightarrow$-calculus is used, that is, redexes that are created during rewriting are also contracted.

To get an idea of what kind of difference in the rewrite relations we have, due to these distinct evaluation mechanisms, consider the following example. The HRS rule $F(\lambda y . z(\lambda x . y x)) \rightarrow z(\lambda x . x)$ with assignment $\sigma: z \mapsto \lambda u . u K$. We have the rewrite step:

$$
\begin{aligned}
F(\lambda y \cdot y K) & =F(\lambda y \cdot(\lambda x \cdot y x) K) \downarrow_{\beta} \\
& =F(\lambda y \cdot(\lambda u \cdot u K)(\lambda x \cdot y x)) \downarrow_{\beta} \\
& =(F(\lambda y \cdot z(\lambda x \cdot y x)))^{\sigma} \downarrow_{\beta} \\
& \rightarrow(z(\lambda x \cdot x))^{\sigma} \downarrow_{\beta} \\
& =(\lambda u \cdot u K) \lambda x \cdot x \downarrow_{\beta} \\
& =(\lambda x \cdot x) K \downarrow_{\beta} \\
& =K
\end{aligned}
$$

Observe how the complete development of the $\lambda$-redexes of the assignment creates a new redex which is also contracted (in the last line). This redex is 'created downwards', so for this process to end, we cannot rely on termination of developments or even superdevelopments (cf. [Raa93]), but really need strong normalisation of simply typed $\lambda$-calculus. On the other hand, the corresponding CRS rule $F([y] Z(y)) \rightarrow Z([x] x)$ and assignment $\sigma: Z \mapsto \underline{\lambda}(u)$.@(u,K), act more lazily:

$$
\begin{aligned}
F([y] @(y, K)) & =(F([y] Z(y)))^{\sigma} \\
& \rightarrow(Z([x] x))^{\sigma} \\
& =@([x] x, K)
\end{aligned}
$$

The substitution is evaluated by a complete development of the $\underline{\lambda}$-redex. We have to add an explicit $\beta$-reduction step, namely

$$
@([x] x, K) \rightarrow K
$$

in order to simulate the HRS rewrite step completely.

An other way of looking at it is to view [-]- really as an abbreviation of $\Lambda(\underline{\lambda}$-.- $)$, for some fresh symbol $\Lambda$. Now, although it seems that $\beta$-redexes can be created in the substitution process above due to the presence of $\underline{\underline{\lambda}}$ 's in terms, this is not the case because they are always 'blocked' by the $\Lambda$. In the example, we end up with the term @ $(\Lambda(\underline{\lambda} x \cdot x), K)$. A 'rule' like @ $\left(\Lambda(Z), Z^{\prime}\right) \rightarrow Z Z^{\prime}$ is needed to 'unblock' the metalanguage redex $(\underline{\lambda} x . x) K$. This is the only thing used in the translation of CRSs into HRSs. 
The same 'blocking' idea of this translation can also be used to show that developments of terms in $\lambda$-calculus must terminate: put fresh variables in front of' abstractions and applications not taking part in a $\beta$-redex. This gives a trivial typable $\lambda \rightarrow$-term which exactly simulates developments. Creating new redexes is prevented by the presence of the fresh variables.

\section{Translating a CRS into a HRS}

In this section we will show that a CRS can be translated into a HRS such that there is a one-to-one correspondence between rewritings in the CRS and in its translation. We use $\left\langle_{-}\right\rangle$as notation for the translation. The mapping $\left\langle_{-}\right\rangle$is chosen to be injective.

Definition 3. The HRS alphabet $\langle\mathcal{A}\rangle$ associated with a CRS alphabet $\mathcal{A}$ consists of

- the symbol $\Lambda:(0 \rightarrow 0) \rightarrow 0$ (meant to 'collapse' a functional type),

- a variable $\langle x\rangle=x: 0$, for each variable $x$ in $\mathcal{A}$,

- a constant $\langle F\rangle=F: 0 \rightarrow \ldots \rightarrow 0 \rightarrow 0(\operatorname{Ar}(F)$ times an $\rightarrow)$, for each operator $F$ in $\mathcal{A}$,

- a variable $\langle Z\rangle=z: 0 \rightarrow \ldots \rightarrow 0 \rightarrow 0(\operatorname{Ar}(Z)$ times an $\rightarrow)$, for each metavariable $Z$ in $\mathcal{A}$,

and the ordinary symbols in a HRS alphabet.

Note that only one base type, namely 0 , is used. The translation between CRS metaterms and contexts is defined by extending the translation of symbols as follows.

Definition 4. (1) $\langle[x] t\rangle=\Lambda(\lambda x .\langle t\rangle)$

Abstractions are translated as projected $\lambda$-abstractions.

(2) $\langle F\rangle=F$ for $F$-ary, and $\left\langle F\left(t_{1}, \ldots, t_{n}\right)\right\rangle=F\left\langle t_{1}\right\rangle \ldots\left\langle t_{n}\right\rangle$ for $F n$-ary with $n \geq 1$

$\langle Z\rangle=z$ for $Z$ 0-ary, and $\left\langle Z\left(t_{1}, \ldots, t_{n}\right)\right\rangle=z\left\langle t_{1}\right\rangle \ldots\left\langle t_{n}\right\rangle$ for $Z n$-ary with $n \geq 1$

Functional terms are translated by currying.

(3) $\langle\square\rangle=\square: 0$

Holes are of base type.

The translation of a context $\langle C[]\rangle$ is denoted by $\langle C\rangle[]$. The translation of CRS rule $R=l \rightarrow r$ is defined as $\langle R\rangle=\langle l\rangle \rightarrow\langle r\rangle$.

For a CRS $\mathcal{C}$, the HRS $\langle\mathcal{C}\rangle$ is obtained by translating the alphabet and the set of rules of $\mathcal{C}$. We often restrict attention to the sub-HRS of $\langle\mathcal{C}\rangle$ where only terms that are translations of terms in $\mathcal{C}$ are considered. We first give the translation of the main ingredient needed in a rewrite step: assignment.

Definition 5. The translation $\langle\sigma\rangle$ of an assignment $\sigma$ is defined as follows: if $\sigma(Z)=\underline{\lambda}\left(x_{1}, \ldots, x_{n}\right) \cdot s$, then $\langle\sigma\rangle(z)=\lambda x_{1} \ldots x_{n} \cdot\langle s\rangle$. 
We now show that these translations are correct in the sense that a CRS concept yields the corresponding HRS concept.

Proposition 6. Let $s^{\prime}$ be the translation $\langle s\rangle$ of a CRS metaterm s. Then

a $s^{\prime}: 0$, moreover there is a bijective correspondence between subterms of $s$ and subterms of type 0 of $s^{\prime}$,

b $s^{\prime}$ is in long $\beta \eta$-normal form,

c if $s$ satisfies the pattern-condition, then $s^{\prime}$ satisfies the pattern-condition,

d $\langle\operatorname{Fvar}(s)\rangle=\operatorname{Fvar}\left(s^{\prime}\right)$, where Fvar denotes the set of free variables and of metavariables in a CRS (or HRS) metaterm.

PROOF. The four properties are proved simultaneously, by structural induction.

The bijective correspondence in a can be made more precise using the notion of position.

Proposition 7. The translations of CRS rules, contexts, and assignments yield the corresponding concepts in the associated HRS.

Next we state some propositions expressing the interaction between forming contexts and applying assignments on the one hand and translating on the other hand.

\section{Proposition 8.}

a $\langle C[t]\rangle=\langle C\rangle[\langle t\rangle]$

b $\left\langle C\left[t_{1}, \ldots, t_{n}\right]\right\rangle=\langle C\rangle\left[\left\langle t_{1}\right\rangle, \ldots,\left\langle t_{n}\right\rangle\right]$

c $\left\langle s\left[x_{1}:=t_{1} \ldots x_{n}:=t_{n}\right]\right\rangle=\langle s\rangle\left[x_{1}:=\left\langle t_{1}\right\rangle \ldots x_{n}:=\left\langle t_{n}\right\rangle\right]$

d $\left\langle t^{\sigma}\right\rangle=\langle t\rangle^{\langle\sigma\rangle} \downarrow_{\beta}$.

Proof.

a The proof proceeds by induction on the structure of $C[]$.

b By repeatedly applying a.

c Choose a context $C[, \ldots$,$] such that s=C\left[x_{i_{1}}, \ldots, x_{i_{j}}\right]$ and precisely the occurrences of the variables $x_{1}, \ldots, x_{n}$ are being displayed. Then

$$
\begin{aligned}
\left\langle s\left[x_{1}:=t_{1} \ldots x_{n}:=t_{n}\right]\right\rangle & =\left\langle C\left[t_{i_{1}}, \ldots, t_{i_{j}}\right]\right\rangle \\
& =\langle C\rangle\left[\left\langle t_{i_{1}}\right\rangle, \ldots,\left\langle t_{i_{j}}\right\rangle\right] \quad \text { (by } \mathbf{b} \text { ) } \\
& =\langle s\rangle\left[x_{1}:=\left\langle t_{1}\right\rangle \ldots x_{n}:=\left\langle t_{n}\right\rangle\right] \text { (use Proposition } 6 \mathbf{a} \text { ) }
\end{aligned}
$$

d The statement is proved by induction on the structure of the metaterm $t$. Note that all $\lambda$ 's in $\langle t\rangle$ that are introduced by translating the abstraction operator do not yield a $\beta$-redex since they are 'blocked' by their big brother $\Lambda$.

Now we show that rewrite steps are naturally preserved by the translation.

Theorem 9. If $s \rightarrow_{R} t$ in a CRS C with $R=l \rightarrow r$ a rewrite rule, then we have $\langle s\rangle \rightarrow\langle R\rangle\langle t\rangle$ in the corresponding $H R S \mathcal{H}$. 
Proof. Let $s \rightarrow\langle R\rangle$ in $\mathcal{C}$, where $s=C\left[l^{\sigma}\right]$ and $t=C\left[r^{\sigma}\right]$, for some context $C[]$ and some assignment $\sigma$. Then,

$$
\begin{array}{rlr}
\langle s\rangle & =\left\langle C\left[l^{\sigma}\right]\right\rangle & \\
& =\langle C\rangle\left[\left\langle l^{\sigma}\right\rangle\right] \quad \quad \text { (by Proposition 8 a) } \\
& =\langle C\rangle\left[\langle l\rangle^{\langle\sigma\rangle} \downarrow_{\beta}\right] \quad(\text { by Proposition 8 d) } \\
& \rightarrow\langle C\rangle\left[\langle r\rangle^{\langle\sigma\rangle} \downarrow_{\beta}\right] & \\
& =\langle C\rangle\left[\left\langle r^{\sigma}\right\rangle\right] & \text { (by Proposition } 8 \mathrm{~d} \text { ) } \\
& =\left\langle C\left[r^{\sigma}\right]\right\rangle & \quad \text { (by Proposition } 8 \mathrm{a}) \\
& =\langle t\rangle &
\end{array}
$$

This proves that for every rewrite step in a CRS $\mathcal{C}$ a rewrite step in the associated HRS $\mathcal{H}$ can be performed. Now we will show that a rewrite step in the translation of a term must originate from a rewrite step in $\mathcal{C}$ itself. For this, we will use that both contexts and assignments in $\mathcal{H}$ can be translated back into the corresponding concepts in $\mathcal{C}$, under the proper restrictions.

\section{Proposition 10.}

a If $C^{\prime}\left[t^{\prime}\right]=\langle s\rangle$, then there exist $C[]$ and $t$ such that $\langle C[]\rangle=C^{\prime}[]$ and $\langle t\rangle=t^{\prime}$.

b If $\langle l\rangle^{\sigma^{\prime}} \downarrow_{\beta}=\langle s\rangle$ and $l$ satisfies the conditions for a left-hand side of a CRS rule, then there exists a $\sigma$ such that $\langle\sigma\rangle=\sigma^{\prime}$.

Proof.

a From Proposition 6 a we have $C^{\prime}[]: 0$ and by the definition of a HRS context $t^{\prime}: 0$. Then the bijective correspondence of Proposition 6 a provides us with suitable $C[]$ and $t$.

b By Proposition $6 \mathrm{~d}$, we know that a free variable $z$ in $\langle l\rangle$ stems from a metavariable $Z$ in $l$. By the pattern-condition, each free variable occurs only in subterms of the form $z x_{1} \ldots x_{n}$ in $\langle l\rangle$, which have type 0 by Proposition $6 \mathrm{a}$, where $n$ is the arity of $Z$. Note that all the $x_{i}$ have type 0 , so this subterm is $\eta$-expanded. If $\sigma^{\prime}(z)=\lambda y_{1} \ldots y_{m} . t^{\prime}$, then $m=n$, because $\sigma^{\prime}(z)$ is by definition in long $\beta \eta$-normal form. Hence, $\left(z x_{1} \ldots x_{n}\right)^{\sigma^{\prime}} \downarrow_{\beta}=t^{\prime}\left[y_{1}:=\right.$ $\left.x_{1} \ldots y_{n}:=x_{n}\right] \downarrow_{\beta}=t^{\prime}\left[y_{1}:=x_{1} \ldots y_{n}:=x_{n}\right]$, because renaming doesn't create redexes. It is easy to show that $t^{\prime}\left[y_{1}:=x_{1} \ldots y_{n}:=x_{n}\right]$ must in fact be a subterm (of type 0 !) of $\langle s\rangle$ and therefore a translation of some term $\hat{t}$. Now we can define $\sigma(Z)=\underline{\lambda}\left(y_{1}, \ldots, y_{n}\right) \cdot \hat{t}\left[x_{1}:=y_{1} \ldots x_{n}:=y_{n}\right]$, which meets the requirements. Note that by the pattern-condition all the $x_{i}$ are distinct.

Theorem 11. Let $\mathcal{C}$ be a $C R S$ and $\mathcal{H}$ its associated HRS. If $\langle s\rangle \rightarrow\langle R\rangle t^{\prime}$ in $\mathcal{H}$ by rewrite rule $\langle R\rangle=\langle l\rangle \rightarrow\langle r\rangle$, then $s \rightarrow_{R} t$ for some CRS term $t$ such that $\langle t\rangle=t^{\prime}$. 
Proof. Let $\langle s\rangle \rightarrow\langle R\rangle t^{\prime}$ in $\mathcal{H}$ with $\langle R\rangle=\langle l\rangle \rightarrow\langle r\rangle$. Then $\langle s\rangle=C^{\prime}\left[\langle l\rangle^{\sigma^{\prime}} \downarrow_{\beta}\right]$ and $t^{\prime}=C^{\prime}\left[\langle r\rangle^{\sigma^{\prime}} \downarrow_{\beta}\right]$ for some context $C^{\prime}[]$ and some assignment $\sigma^{\prime}$. Then

$$
\begin{aligned}
& \langle s\rangle=C^{\prime}\left[\langle l\rangle^{\sigma^{\prime}} \downarrow_{\beta}\right] \\
& =\langle C\rangle\left[\langle l\rangle^{\sigma^{\prime}} \downarrow_{\beta}\right] \text { (by Proposition } 10 \mathrm{a} \text { ) } \\
& =\langle C\rangle\left[\langle l\rangle^{\left\langle\sigma \downarrow_{\beta}\right.}\right] \text { (by Proposition } 10 \mathrm{~b} \text { ) } \\
& =\langle C\rangle\left[\left\langle l^{\sigma}\right\rangle\right] \quad \text { (by Proposition } 8 \mathrm{~d} \text { ) } \\
& =\left\langle C\left[l^{\sigma}\right]\right\rangle \quad \text { (by Proposition } 8 \text { a) }
\end{aligned}
$$

By injectivity we have $s=C\left[l^{\sigma}\right]$. If we take $t=C\left[r^{\sigma}\right]$, then $s \rightarrow t$ and

$$
\begin{aligned}
\langle t\rangle & =\left\langle C\left[r^{\sigma}\right]\right\rangle \\
& =\langle C\rangle\left[\left\langle r^{\sigma}\right\rangle\right] \quad \text { (by Proposition 8 a) } \\
& =\langle C\rangle\left[\langle r\rangle^{\langle\sigma\rangle} \downarrow_{\beta}\right] \quad(\text { by Proposition 8 d) } \\
& =\langle C\rangle\left[\langle r\rangle^{\sigma^{\prime}} \downarrow_{\beta}\right] \\
& =t^{\prime}
\end{aligned}
$$

The naturality of a translation is can be measured by the properties which it preserves and reflects. Theorems 9 and 11 state that the main property of CRSs and HRSs, i.e. whether one term rewrites (in one step) to another, is both preserved and reflected. Combining this with the fact that orthogonality is preserved, we obtain a confluence proof for orthogonal CRSs via confluence of their associated HRS. A system is orthogonal if it is left-linear and nonambiguous. For a precise definition of orthogonality we refer the reader to [Klo80] and $[\mathrm{Nipb}]$.

Corollary 12. Orthogonal CRSs are confluent.

PRoof. Let $s \rightarrow C t_{1}$ and $s \rightarrow \mathcal{C} t_{2}$ be rewrites in an orthogonal CRS $\mathcal{C}$. By Theorem 9, we can lift these to rewrites $\langle s\rangle \rightarrow \mathcal{H}\left\langle t_{1}\right\rangle$ and $\langle s\rangle \rightarrow \mathcal{H}\left\langle t_{2}\right\rangle$ in the HRS $\mathcal{H}$ associated to $\mathcal{C}$. Because $\mathcal{H}$ is easily seen to be orthogonal, we conclude from [Nipb, Cor. 4.9] that it is confluent, hence there exist rewrites $\left\langle t_{1}\right\rangle \rightarrow \mathcal{H} r^{\prime}$ and $\left\langle t_{2}\right\rangle \rightarrow \mathcal{H} r^{\prime}$, for some $r^{\prime}$. These sequences can be projected again to form $t_{1} \rightarrow \mathcal{C} r$ and $t_{2} \rightarrow_{\mathcal{C}} r$ by Theorem 11, also showing that $\langle r\rangle=r^{\prime}$. The proof is expressed by the following diagram. 


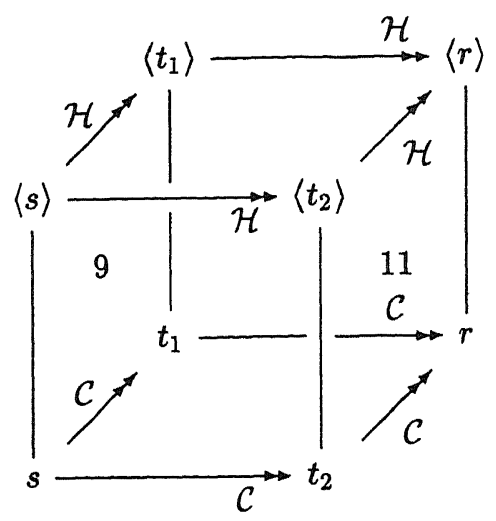

\section{Translating a HRS into a CRS plus explicit $\beta$}

In this section we define a translation from HRSs into CRSs. This translation is not as straightforward as the one the other way round, due to the fact that the metalanguage of HRSs, $\lambda \rightarrow$, has more 'rewrite power' than the (hidden) metalanguage of CRSs, $\underline{\lambda}$. In order to be able to simulate every rewrite of a HRS in its associated CRS, a $\beta$-reduction rule and a binary symbol @ for application have to be added to the translation. It is given as

$$
@\left([x] Z(x), Z^{\prime}\right) \rightarrow_{\beta} Z\left(Z^{\prime}\right)
$$

We will denote $\beta$-reduction to normal form as $\rightarrow{ }_{\beta}^{\prime}$. To simplify the notation a bit we sometimes use $@_{n}$ to abbreviate $n$ applications, for instance $@_{2}(A, B, C)$ stands for @(@ $(A, B), C)$. Formally, $@_{n}$ for $n \geq 1$ is defined as

$$
\begin{aligned}
@_{1}\left(t, t_{1}\right) & =@\left(t, t_{1}\right) \\
@_{n+1}\left(t, t_{1}, \ldots, t_{n}, t_{n+1}\right) & =@\left(@_{n}\left(t, t_{1}, \ldots, t_{n}\right), t_{n+1}\right)
\end{aligned}
$$

Again, the translation is denoted as $\langle-\rangle$ and is chosen to be injective. We do not obtain a 1 -1-correspondence between rewrite steps in a HRS and rewrite steps in its encoding, but the translation does satisfy a weaker property. Let $\mathcal{H}$ be a HRS and let $\mathcal{C}$ be its encoding, having as rewrite rules the translated rules of $\mathcal{H}$ and the $\beta$-rule. We have that if $s \rightarrow_{\mathcal{H}} t$ in a HRS $\mathcal{H}$, then $\langle s\rangle \rightarrow_{\mathcal{C} \beta}\langle t\rangle$, where $\rightarrow \mathcal{C}_{\beta}$ is defined to be a rewrite in $\mathcal{C}$ consisting of one step via a translated $\mathcal{H}$-rule followed by a $\beta$-reduction to $\beta$-normal form. Moreover, we obtain that a rewrite in the encoding of $\mathcal{H}$ starting with the encoding of some term of $\mathcal{H}$ can be extended to a rewrite corresponding to a rewrite in the original HRS.

First to an alphabet $\mathcal{A}$ of a HRS a CRS alphabet $\langle\mathcal{A}\rangle$ is associated.

Definition 13. The CRS alphabet $\langle\mathcal{A}\rangle$ associated with a HRS alphabet $\mathcal{A}$ consists of 
- a symbol @ for application,

- for every symbol $F \in \mathcal{A}$ for an operator of type $\tau$, a symbol $F$ for an operator with arity $n=\operatorname{Ar}(\tau)$

- the ordinary symbols of a CRS alphabet, i.e. symbols for variables $x y z \ldots$, symbols for metavariables with a fixed arity $Z Z_{0} Z_{1} \ldots$ and a symbol for abstraction, [-].

Definition 14. The translation of terms in long $\beta \eta$-normal form is defined inductively as follows:

$$
\begin{aligned}
&\left\langle\lambda x_{1} \ldots x_{m} . x t_{1} \ldots t_{n}\right\rangle= \begin{cases}{\left[x_{1}\right] \ldots\left[x_{n}\right] x} & \text { if } n=0 \\
{\left[x_{1}\right] \ldots\left[x_{n}\right] @_{n}\left(x,\left\langle t_{1}\right\rangle, \ldots,\left\langle t_{n}\right\rangle\right)} & \text { if } n \geq 1\end{cases} \\
& \text { - }\left\langle\lambda x_{1} \ldots x_{m} . F t_{1} \ldots t_{n}\right\rangle= \begin{cases}{\left[x_{1}\right] \ldots\left[x_{n}\right] F} & \text { if } n=0 \\
{\left[x_{1}\right] \ldots\left[x_{n}\right] F\left(\left\langle t_{1}\right\rangle, \ldots,\left\langle t_{n}\right\rangle\right)} & \text { if } n \geq 1\end{cases}
\end{aligned}
$$

It is extended to contexts by defining $\langle\square\rangle=\square$. We write $\langle C\rangle[]$ for the translation of $C[]$.

Free variables in terms of HRSs correspond to free variables in terms of CRSs. Free variables in rules of HRSs correspond to metavariables in rules of CRSs. Therefore a separate definition of the translation of a rule has to be given, in which free variables are translated in another way than in the translation of a term.

Definition 15. The translation $\langle l \rightarrow r\rangle$ of a HRS rule $l \rightarrow r$ is defined as $\langle l\rangle \rightarrow\langle r\rangle$, where $\langle l\rangle$ and $\langle\boldsymbol{r}\rangle$ are defined inductively as follows.

a The left-hand side $l$ of a HRS rewrite rule is of the form $l=F t_{1} \ldots t_{k}$. Here $t_{1}, \ldots, t_{k}$ are long $\beta \eta$-normal forms in which inputs of free variables are $\eta$ equivalent to distinct bound variables. The translation $\langle l\rangle$ of $l$ is defined by induction on the structure of such a long $\beta \eta$-normal form.
- $\left\langle\lambda x_{1} \ldots x_{m} \cdot x t_{1} \ldots t_{n}\right\rangle= \begin{cases}{\left[x_{1} \ldots x_{m}\right] x} & \text { if } n=0 \\ {\left[x_{1} \ldots x_{m}\right] @_{n}\left(x,\left\langle t_{1}\right\rangle, \ldots,\left\langle t_{n}\right\rangle\right)} & \text { if } n \geq 1\end{cases}$
- $\left\langle\lambda x_{1} \ldots x_{m} . z t_{1} \ldots t_{n}\right\rangle= \begin{cases}{\left[x_{1} \ldots x_{m}\right] Z} & \text { if } n=0 \\ {\left[x_{1} \ldots x_{m}\right] Z\left(t_{1} \downarrow_{\eta}, \ldots, t_{n} \downarrow_{\eta}\right)} & \text { if } n \geq 1\end{cases}$

if $z$ is a variable which is free in $l$ (note that $t_{1}, \ldots, t_{n}$ are $\eta$-equivalent to distinct bound variables by the pattern-condition),

$$
\text { - }\left\langle\lambda x_{1} \ldots x_{m} . F t_{1} \ldots t_{n}\right\rangle= \begin{cases}{\left[x_{1} \ldots x_{m}\right] F} & \text { if } n=0 \\ {\left[x_{1} \ldots x_{m}\right] F\left(\left\langle t_{1}\right\rangle, \ldots,\left\langle t_{n}\right\rangle\right)} & \text { if } n \geq 1\end{cases}
$$

b The right-hand side $r$ of a HRS rewrite rule is of the form $r=s t_{1} \ldots t_{k}$ with $s$ a symbol standing for a free variable or an operator and $t_{1}, \ldots, t_{k}$ in long $\beta \eta$-normal form. The translation $\langle r\rangle$ of $r$ is by induction on the structure of a long $\beta \eta$-normal form.

$$
\begin{aligned}
\left\langle\lambda x_{1} \ldots x_{m} . x t_{1} \ldots t_{n}\right\rangle & = \begin{cases}{\left[x_{1} \ldots x_{m}\right] x} & \text { if } n=0 \\
{\left[x_{1} \ldots x_{m}\right] @_{n}\left(x,\left\langle t_{1}\right\rangle, \ldots,\left\langle t_{n}\right\rangle\right)} & \text { if } n \geq 1\end{cases} \\
\text { - }\left\langle\lambda x_{1} \ldots x_{m} . z t_{1} \ldots t_{n}\right\rangle & = \begin{cases}{\left[x_{1} \ldots x_{m}\right] Z} & \text { if } n=0 \\
{\left[x_{1} \ldots x_{m}\right] Z\left(\left\langle t_{1}\right\rangle, \ldots,\left\langle t_{n}\right\rangle\right)} & \text { if } n \geq 1\end{cases} \\
\text { - } n & \left\langle\lambda x_{1} \ldots x_{m} . F t_{1} \ldots t_{n}\right\rangle= \begin{cases}{\left[x_{1} \ldots x_{m}\right] F} & \text { if } n=0 \\
{\left[x_{1} \ldots x_{m}\right] F\left(\left\langle t_{1}\right\rangle, \ldots,\left\langle t_{n}\right\rangle\right)} & \text { if } n \geq 1\end{cases}
\end{aligned}
$$


As in the translation from CRSs to HRSs, we show that rewrite steps in a HRS can be simulated by essentially the same step in the associated CRS. To that end, the translation is extended to assignments.

Definition 16. An assignment of a HRS assigns to a variable a term in long $\beta \eta$ normal form of the same type. So an assignment assigns to a variable $y$ of type $\tau$ a term of the form $\lambda x_{1} \ldots x_{n} . t$ with $n=\operatorname{Ar}(\tau)$ and $t$ not a $\lambda$-abstraction. The translation $\langle\sigma\rangle$ of an assignment $\sigma$ is defined as follows: if $\sigma(y)=\lambda x_{1} \ldots x_{n} . t$, then $\langle\sigma\rangle(Y)=\underline{\lambda}\left(x_{1}, \ldots, x_{n}\right),\langle t\rangle$.

First we show that the translation produces correct terms and that the translation of a rewrite rule is well-defined.

\section{Proposition 17.}

a If $t$ is a HRS term in long $\beta \eta$-normal form, then $\langle t\rangle$ is well-defined as a CRS term.

b The translation $\langle l \rightarrow r\rangle$ of a HRS rewrite rule $l \rightarrow r$ satisfies the definition of a CRS rewrite rule.

Then we show that decomposing a term by a context, commutes with the translation.

Proposition 18. $\langle C[t]\rangle=\langle C\rangle[\langle t\rangle]$.

Proof. The proof proceeds by induction on the definition of $C[]$.

Finally we show that decomposing a term into a (meta)term and an assignment almost commutes with the translation. For a decomposition into a left-hand side, which is a pattern, the commutation is perfect, but for right-hand sides we need additional $\beta$-steps. This is proved in the following two propositions.

\section{Proposition 19.}

a Let $s$ be a term in long $\beta \eta$-normal form, and $u_{1}, \ldots, u_{n}$ terms that are $\eta$-equivalent to distinct variables. Then $\langle s\rangle\left[z_{1}:=u_{1} \downarrow_{\eta} \ldots z_{n}:=u_{n} \downarrow_{\eta}\right]=$ $\left\langle s\left[z_{1}:=u_{1} \ldots z_{n}:=u_{n}\right] \downarrow_{\beta}\right\rangle$.

b $\langle l\rangle^{\langle\sigma\rangle}=\left\langle l^{\sigma} \downarrow_{\beta}\right\rangle$ for $l$ a term in long $\beta \eta$-normal form satisfying the pattern condition.

Proof.

a The proof proceeds by induction on the structure of $s$.

b The statement is proved by induction on the structure of a long $\beta \eta$-normal form, in which arguments of free variables are $\eta$-equivalent to distinct bound variables.

Combining the last two propositions, we observe that the 'matching power' (or complexity of matching, depending on one's point of view) of HRSs is equally present in CRSs, making a natural encoding of the former into the latter possible. This is due to the pattern-condition of HRSs. For HRSs not satisfying the 
pattern-condition (cf. [Wol93]) this is no longer the case, and an encoding doesn't seem to be straightforward anymore, even if we would lift some of the restrictions on left-hand sides of CRS-rules. The next proposition shows that although CRSs and HRSs have the same matching power, HRSs have more 'rewrite power', i.e. they can do more in one step.

\section{Proposition 20.}

a Let $s$ and $u_{1}, \ldots, u_{n}$ be terms in long $\beta \eta$-normal form. Then we have $\langle s\rangle\left[z_{1}:=\right.$ $\left.\left\langle u_{1}\right\rangle \ldots z_{n}:=\left\langle u_{n}\right\rangle\right] \rightarrow{ }_{\beta}^{\prime}$

$\left\langle s\left[z_{1}:=u_{1} \ldots z_{n}:=u_{n}\right] \downarrow_{\beta}\right\rangle$.

b $\langle r\rangle^{\langle\sigma\rangle} \rightarrow !{ }_{\beta}\left\langle r^{\sigma} \downarrow_{\beta}\right\rangle$.

ProOF.

a The proof proceeds by induction on the maximal length of the $\beta$-reduction of $s\left[z_{1}:=u_{1} \ldots z_{1}:=u_{n}\right]$ to normal form.

b The proof proceeds by induction on the structure of $r$.

Now we can collect the results of this section to show that every rewrite step in a HRS $\mathcal{H}$ can be simulated in its corresponding CRS $\mathcal{C}$.

Theorem 21. If $s \rightarrow_{R} t$ by rewrite rule $R=l \rightarrow r$ in $\mathcal{H}$, then we have $\langle s\rangle \rightarrow_{\langle R\rangle}$ $\rightarrow !{ }_{\beta}\langle t\rangle$, or $\langle s\rangle \rightarrow_{\mathcal{C} \beta}\langle t\rangle$, in the corresponding CRS C.

Proof. The term $s$ is of the form $C\left[l^{\sigma} \downarrow_{\beta}\right]$, and we have $s=C\left[l^{\sigma} \downarrow_{\beta}\right] \rightarrow_{R}$ $C\left[r^{\sigma} \downarrow_{\beta}\right]=t$. We have

$$
\begin{aligned}
& \langle s\rangle=\left\langle C\left[l^{\sigma} \downarrow_{\beta}\right]\right\rangle \\
& =\langle C\rangle\left[\left\langle l^{\sigma} \downarrow_{\beta}\right\rangle\right] \quad \text { (by Proposition 18) } \\
& =\langle C\rangle\left[\langle l\rangle^{\langle\sigma\rangle}\right] \text { (by Proposition } 19 \mathrm{~b} \text { ) } \\
& \rightarrow\langle R\rangle\langle C\rangle\left[\langle r\rangle^{\langle\sigma\rangle}\right] \\
& \rightarrow{ }_{\beta}^{!}\langle C\rangle\left[\left\langle r^{\sigma} \downarrow_{\beta}\right\rangle\right] \text { (by Proposition } 20 \mathbf{b} \text { ) } \\
& =\left\langle C\left[r^{\sigma} \downarrow_{\beta}\right]\right\rangle \quad \text { (by Proposition 18) } \\
& =\langle t\rangle
\end{aligned}
$$

The next thing to be done is to connect somehow a rewrite step in the translation of a HRS with a rewrite step in the original HRS itself. Since the translation of a HRS $\mathcal{H}$ acts as a refinement of $\mathcal{H}$, we cannot hope for a result as neat as in the previous section. But still something can be said. First we will show that if we have the rewrite $\langle s\rangle \rightarrow_{c \beta} t^{\prime}$ in the translation of a HRS, then we can project it to a rewrite step $s \rightarrow \mathcal{H} t$, such that $\langle t\rangle=t^{\prime}$.

The first observation we need is that there is a 1-1 correspondence between functional subterms in $\langle s\rangle$, i.e. subterms with a function symbol (also taking the $@_{n}$ for $n \geq 1$ into account) or a variable as head, and subterms of type 0 in s. Further, we need two propositions. 
Proposition 22. Suppose $C^{\prime}\left[t^{\prime}\right]=\langle s\rangle$ with $t^{\prime}$ a functional term. Then a context $C[]$ and a term $t$ exist such that $\langle C\rangle[]=C^{\prime}[]$ and $\langle t\rangle=t^{\prime}$.

Proof. Via the correspondence we obtain an appropriate subterm of $s$, i.e. a context $C[]$ and a term $t$ such that $s=C[t]$ and $\langle t\rangle=t^{\prime}$. Using Proposition 18 we have that $\langle C\rangle[]=C^{\prime}[]$.

Proposition 23. Let $l$ be the left-hand side of a HRS rewrite rule. If $\langle l\rangle^{\sigma^{\prime}}=\langle s\rangle$, then there is an assignment $\sigma$ with $\langle\sigma\rangle=\sigma^{\prime}$.

Proof. Metavariables $Z_{i}$ occur in $\langle l\rangle$ in the form $Z\left(x_{1}, \ldots x_{n}\right)$. Suppose $\sigma^{\prime}$ is defined as $\sigma^{\prime}\left(Z_{i}\right)=\underline{\lambda}\left(u_{1}, \ldots, u_{n}\right) \cdot t^{\prime}$, hence $\left(Z\left(x_{1}, \ldots x_{n}\right)\right)^{\sigma^{\prime}}=t^{\prime}\left[u_{1}:=x_{1} \ldots u_{n}:=\right.$ $\left.x_{n}\right]$. We know that $t^{\prime} \neq[x] t^{\prime \prime}$, because otherwise we cannot have $\langle l\rangle^{\sigma^{\prime}}=\langle s\rangle$ due to the typing. Hence, $t^{\prime}[\ldots]$ is a functional term, i.e. of one of the forms $F(\ldots)$ or $@_{n}(\ldots)$ with $n \geq 1$ and so in $s$ there is a corresponding subterm $t$ of type 0 , such that $\langle t\rangle=t^{\prime}\left[u_{1}:=x_{1} \ldots u_{n}:=x_{n}\right]$. Define $\sigma$ as $\sigma z=\lambda x_{1} \ldots x_{n} . t$. Then $\langle\sigma\rangle=\sigma^{\prime}$.

Theorem 24. If $\langle s\rangle \rightarrow\langle R\rangle \rightarrow{ }_{\beta}^{\prime} t^{\prime}$ in the CRS $\mathcal{C}$ by rewrite rule $\langle R\rangle=\langle l \rightarrow r\rangle$, then $s \rightarrow_{R} t$ in $\mathcal{H}$, for some $t$ such that $\langle t\rangle=t^{\prime}$.

Proof. We have $\langle s\rangle=C^{\prime}\left[\langle l\rangle^{\sigma^{\prime}}\right] \rightarrow c t_{0}=C^{\prime}\left[\langle r\rangle^{\sigma^{\prime}}\right] \rightarrow{ }_{\beta}^{!} t^{\prime}$, for some context $C^{\prime}[]$, assignment $\sigma^{\prime}$. Now we have

$$
\begin{aligned}
& \langle s\rangle=C^{\prime}\left[\langle l\rangle^{\sigma^{\prime}}\right] \\
& =\langle C\rangle\left[\langle l\rangle^{\sigma^{\prime}}\right] \quad \text { (by Proposition 22) } \\
& =\langle C\rangle\left[\langle l\rangle^{\langle\sigma\rangle}\right] \quad \text { (by Proposition 23) } \\
& =\langle C\rangle\left[\left\langle l^{\sigma} \downarrow_{\beta}\right\rangle\right] \text { (by Proposition } 19 \mathbf{b} \text { ) } \\
& =\left\langle C\left[l^{\sigma} \downarrow_{\beta}\right]\right\rangle \quad \text { (by Proposition 18) }
\end{aligned}
$$

By injectivity of $\left\langle_{-}\right\rangle$, we have $s=C\left[l^{\sigma} \downarrow_{\beta}\right]$. Take $t=C\left[r^{\sigma} \downarrow_{\beta}\right]$, then $s \rightarrow_{R} t$ and

$$
\begin{aligned}
\langle t\rangle & =\left\langle C\left[r^{\sigma} \downarrow_{\beta}\right]\right\rangle \\
& =\langle C\rangle\left[\left\langle r^{\sigma} \downarrow_{\beta}\right\rangle\right] \quad \text { (by Proposition 18) } \\
& \leftarrow_{\beta}^{!}\langle C\rangle\left[\langle r\rangle^{\langle\sigma\rangle}\right] \text { (by Proposition 20 b) } \\
& =\langle C\rangle\left[\langle r\rangle^{\sigma^{\prime}}\right] \\
& =t_{0}
\end{aligned}
$$

By confluence of $\beta$ and injectivity of $\left\langle_{-}\right\rangle$, we have $\langle t\rangle=t^{\prime}$.

If we want to prove the Church-Rosser property for orthogonal HRSs via the same property for CRSs, Theorem 24 is not quite enough. The $\beta$-rule, by construction, indeed is orthogonal to the other rules and coinitial rewrites can be lifted, but only $\mathcal{C} \beta$-steps can be projected, not arbitrary $\mathcal{C}$-rewrites. We now 
show that every rewrite in an arbitrary CRS $\mathcal{C}$ starting with a term which is the translation of some HRS-term, can be completed, by performing a $\beta$-reduction to $\beta$-normal form, to a rewrite which can be simulated by a 'standard' rewrite consisting of $\mathcal{C} \beta$-steps.

The proof follows the strategy employed for proving $\Sigma \models \mathrm{WCR}^{+}$in $[\mathrm{Klo} 80$, pp. 144-148]. However, some difficulties arise. First, because of the possible nonleft-linearity of the rules. Second, because simply typed $\lambda$-calculus doesn't satisfy the disjointness property in contrast to underlined $\lambda$-calculus.

The main property to be proved is that $\beta$-reductions to normal form do not interfere with rewrite steps. To do this we first need to define some tracing mechanisms.

\section{Definition 25.}

a Let $R=l \rightarrow r$ be a rewrite rule. Its conditional version $R_{c}=l_{c} \rightarrow r$ is obtained by repeatedly replacing occurrences of a metavariable $Z$ which occurs at least twice in $l$ by a fresh metavariable $Z^{\prime}$ and adding the condition $Z \equiv Z^{\prime}$ to the rule. Its linearisation is $R_{l}$ obtained from $R_{c}$ by omitting the conditions.

b Let $r$ be a metaterm, and $\mathbf{Z}=Z_{1}, \ldots, Z_{n}$ be a list of metavariables containing the ones in $r$, then the freezing $r_{f}$ of $\mathbf{Z}$ in $r$ is defined by $r_{f}=$ $@_{n}\left([\mathbf{z}] r^{\prime},\left[\mathbf{x}_{1}\right] Z_{1}\left(\mathbf{x}_{1}\right), \ldots\left[\mathbf{x}_{\mathbf{n}}\right] Z_{n}\left(\mathbf{x}_{\mathbf{n}}\right)\right)$, where $\mathbf{z}=z_{1}, \ldots, z_{n}$ is a list of fresh variables, and $r^{\prime}$ is obtained by replacing in $r$ all occurrences of $Z(\mathrm{t})$ by $@(z, \mathbf{t})$. For a rule $R=l \rightarrow r$, the freezing $R_{f}=l \rightarrow r_{f}$ is defined by freezing the metavariables of $l$ in $r$. We define $R_{f \beta}$ to be the CRS with rules $R_{f}$ and $\beta$

c Let $R=l \rightarrow r$ be a rewrite rule. Its underlining $\underline{R}$ is obtained from $R_{c f}$ by underlining the head-symbol of $l_{c}$. (So $R$ is first made conditional, then frozen and finally its head-symbol is underlined.)

d An ( $R$-)underlining of a term $s$ is a term containing some underlined symbols, which are the head-symbols of $\underline{R}$-redexes, and which is equal to $s$ after removing the underlining.

e A rewrite $s \rightarrow_{\mathcal{C}} t$ is an (R-)development if there is some underlining $\underline{s}$ of $s$, such that $\underline{s} \rightarrow \underline{R}+\beta \underline{t}$ and the underlined rewrite 'projects' onto the original one. Note that, due to non-left-linearity, the terms in the underlined rewrite need not be underlinings of terms.

Notation. This underlining of (head symbols of) redexes might be be considered confusing, because underlinings were also used in $\underline{\lambda}$-calculus. Yet, we think it is the right notation because the underlinings express the same idea of marking both times.

Example 5. Let $R$ be a rule defined by $R=\nu([x] Z(x),[x] Z(x)) \rightarrow Z(\nu([x] Z(x)))$. Then we have

$$
\begin{aligned}
R_{l} & =\nu\left([x] Z(x),[x] Z^{\prime}(x)\right) \rightarrow Z(\nu[x] Z(x)) \\
R_{f} & =\nu([x] Z(x),[x] Z(x)) \rightarrow @([z] @(z, \nu[x] @(z, x)),[x] Z(x)) \\
\underline{R} & =\underline{\nu}\left([x] Z(x),[x] Z^{\prime}(x)\right) \rightarrow @\left(\left[z, z^{\prime}\right] @(z, \nu[x] @(z, x)),[x] Z(x),[x] Z^{\prime}(x)\right) \text { if } Z \equiv Z^{\prime}
\end{aligned}
$$


The idea of freezing is the one of [Lan93], postponing both duplication of the metavariables and substitution into the metavariables. It is more extensive than the one in [Klo80], where only substitution is postponed. Both postponed actions can be performed by $\beta$-reduction:

\section{Proposition 26.}

a $s \rightarrow c_{\beta} t$ is a development.

b Let $\underline{s}$ be equal to $s$ after removal of underlinings. If $\underline{s} \rightarrow_{\underline{R} \beta} \underline{t}$, then $s \rightarrow c \beta t$. Here $\rightarrow \underline{R} \beta=\rightarrow \underline{R} \rightarrow !$.

Proof.

a Idea. Underline the redex to obtain an underlining of $s$. Rewrite it with the underlined rule and then to $\beta$-nf.

b Idea. In the $\beta$-reduction to normal form, we can do the postponed duplication and substitution steps first and then the others. This rewrite can be projected to a $\mathcal{C} \beta$-step.

It is not difficult to see that explicit $\beta$-reductions in translated terms can be made to correspond to $\beta$-reductions in $\lambda^{\rightarrow}$-calculus. (Define a suitable forgetful map, forgetting explicit @'s and replacing [-] by $\lambda$, giving typable terms). Hence $\beta$ is terminating. In the following we only consider rewrites that start with the translation of some term in the HRS.

Proposition 27. Every rewrite in $\underline{R}+\beta$ terminates.

Proof. Sketch. Let $\underline{R}=\underline{l} \rightarrow \underline{r}$. Let $\underline{s}^{\prime}$ be obtained from $\underline{s}$ by replacing all $\underline{R}$ redexes $\underline{l}^{\sigma}$ by $@\left([x] x, \underline{r}^{\sigma}\right)$. This can be done unambiguously because $\underline{R}$ doesn't have overlap with itself. The idea is that we have replaced left-hand sides by their right-hand sides in advance, but have put an extra identity in, to keep in mind that we have to do soto simulate a step. (This replacement works only because the rule is (left- and right-)linear). Now we have that every rewrite starting from $\underline{s}$ can be simulated by a rewrite of the same length starting from $\underline{s}^{\prime}$. More precisely, each $\beta$-step is simulated by a $\beta$-step and an $\underline{R}$-step $C\left[\underline{l}^{\sigma}\right] \rightarrow_{\underline{R}} C\left[\underline{\underline{r}}^{\sigma}\right]$ is simulated by the corresponding $\beta$-step $C^{\prime}\left[@\left([x] x, \underline{r}^{\sigma^{\prime}}\right)\right] \rightarrow_{\beta} C^{\prime}\left[\underline{r}^{\sigma^{\prime}}\right]$. Since $\beta$ rewriting terminates, the $\underline{R}+\beta$-rewrite must be finite.

Corollary 28. Every development is finite.

Proposition 29. $\beta$ commutes with $\underline{R}+\beta$. That is

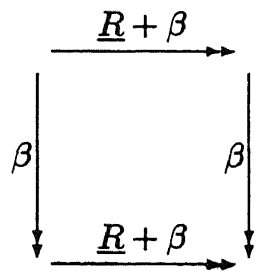


Proof. By termination of $\underline{R}+\beta$ (Proposition 27) and Newman's Lemma (see e.g. [Oos94a]) it suffices to consider only local divergences in proving commutativity. The case of a local divergence of $\beta$-steps is covered by confluence of $\beta$. The other case follows by considering the relative positions of the $\beta$ - and $\underline{R}$-redex. If the $\beta$-redex is inside the $\underline{R}$-redex, we have the following diagram.

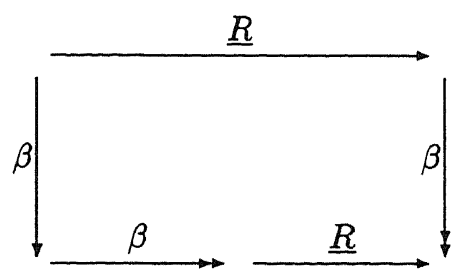

We may need some 'compensating' $\beta$-steps due to possible conditions on $\underline{R}$ (which originate from non-left-linearity of $R$ ). If the $\beta$-redex is outside the $\underline{R}$ redex, we have the diagram:

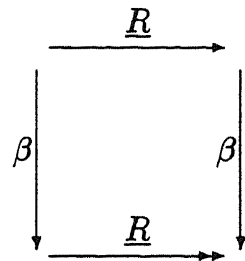

The $\beta$-step may duplicate and even nest $\underline{R}$-redexes, but this does not cause much trouble. A parallel inside-out reduction of $\underline{R}$-redexes works. (This is just as easy as for combinations of $\lambda$-calculus and first-order rewrite systems, as $\underline{R}$ is linear.)

Proposition 30. If $s \rightarrow c_{\beta}$ t and $s \rightarrow ! s^{\prime}$, then $s^{\prime} \rightarrow c_{\beta} t$.

Proof. By Proposition 26 we can construct the reduction $\underline{s} \rightarrow \underline{R} \rightarrow{ }_{\beta}^{!} t$, for some rule $R$ and underlining $\underline{s}$ of $s$. We prove that if $\underline{s} \rightarrow \underline{R} \rightarrow \underline{R}+\beta$, such that $t$ is in $\beta$-normal form, and $\underline{s} \rightarrow{ }_{\beta}^{\prime} \underline{s}^{\prime}$, then $\underline{s}^{\prime} \rightarrow \underline{R} \beta$. The proof is by induction on the maximal length of a $\underline{R}+\beta$ reduction sequence starting from $\underline{s}$ and expressed by the following diagram.

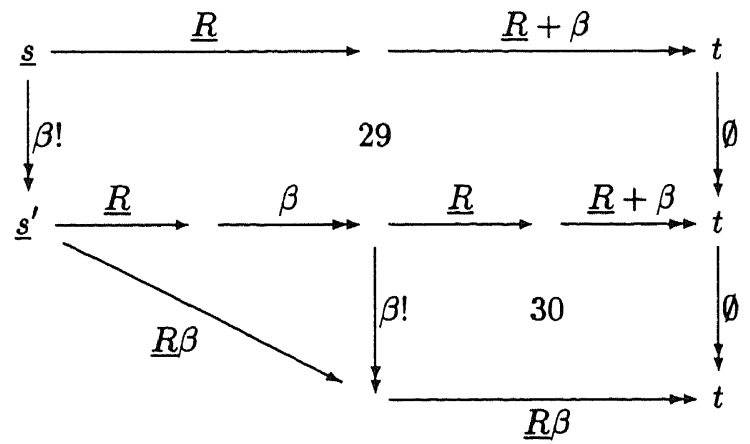

By applying Proposition 26 again (in the reverse direction), we are done. 
Lemma 31. Suppose $s \rightarrow_{\mathcal{C}} t$, and $s$ and $t$ are in $\beta$-normal form, then $s \rightarrow c \beta t$.

PROoF. The proof is expressed by the following diagram

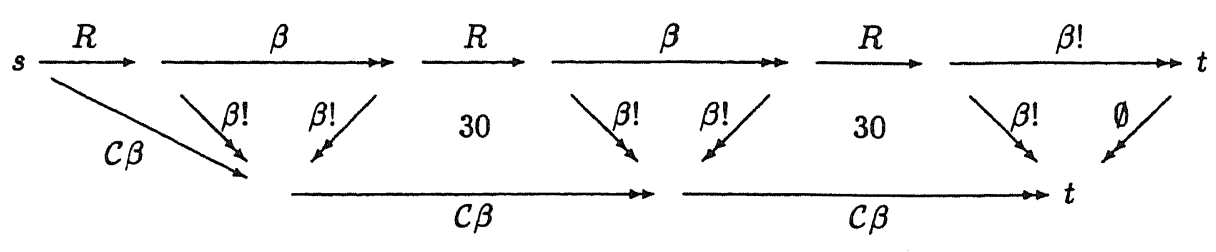

Remark. The results in the literature on modularity of confluence for combinations of typed $\lambda$-calculi with various kinds of rewriting do not seem to apply here. This is because the rewrite rules are not first-order. They can be frozen as above into a 'first-order part' and a 'substitution part', but the former may contain rules with $\beta$-redexes on their right-hand sides, which is not allowed for the systems studied in the literature. On the other hand, the method employed here seems to be quite flexible, since it makes use only of completeness of (typed) $\beta$. For example, the confluence result of [BTG89] should be an easy consequence.

In [Oos94b] a technique which is related to the one in this section is used to prove a Finite Developments Theorem for the large class of orthogonal Higher-Order Rewriting Systems.

Corollary 32. Orthogonal HRSs are confluent.

PRoOF. Suppose we have two coinitial rewrites, $s \rightarrow \mathcal{H} t_{1}$ and $s \rightarrow \mathcal{H} t_{2}$, we can lift them by Theorem 21 to rewrites $\langle s\rangle \rightarrow_{\mathcal{C} \beta}\left\langle t_{1}\right\rangle$ and $\langle s\rangle \rightarrow_{C \beta}\left\langle t_{2}\right\rangle$. Because $\mathcal{C}$ is an orthogonal CRS we can find by [Klo80, Thm. II.3.11] (or [Raa93]), convergent rewrites $\left\langle t_{1}\right\rangle \rightarrow_{C} t^{\prime}$ and $\left\langle t_{2}\right\rangle \rightarrow_{c} t^{\prime}$, for some $t^{\prime}$. If we reduce $t^{\prime}$ to $\beta$-normal form $r^{\prime}$, then Lemma 31 says that the resulting rewrites can be simulated by rewrites $\left\langle t_{1}\right\rangle \rightarrow_{\mathcal{C} \beta} r^{\prime}$ and $\left\langle t_{2}\right\rangle \rightarrow_{C \beta} r^{\prime}$. Finally, by Theorem 24 we can construct rewrites $t_{1} \rightarrow \mathcal{H} r$ and $t_{2} \rightarrow \mathcal{H} r$, such that $\langle r\rangle=r^{\prime}$.

The proof is expressed by the following diagram. 


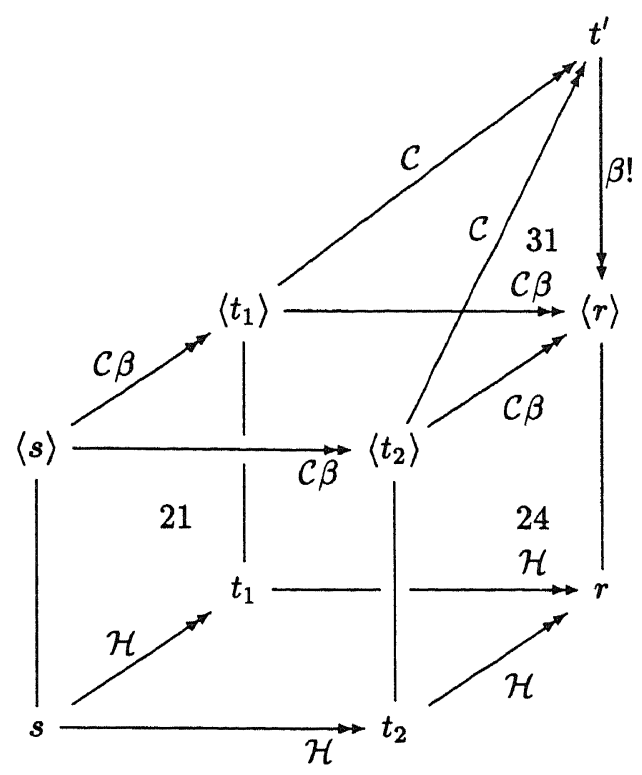

In fact, the reduction sequence $t^{\prime} \rightarrow !{ }_{\beta}\langle r\rangle$, can be shown to be empty, giving a somewhat stronger result.

\section{Discussion}

In the picture below we show the relationships between some classes of rewriting systems occurring in the literature. We have classified them along two dimensions. Horizontally, we distinguish between logical and combinatorial systems. The logical systems are the ones for which the Curry-Howard Isomorphism still makes sense, i.e. the left-hand sides of rules satisfy a constructor-destructor discipline. If the left-hand sides consist of possibly complex combinations of symbols, then we call the system combinatorial. Vertically, we distinguish between firstorder and higher-order in the usual way. One could add a third dimension: functional versus communicational. Apart from the IINs which generalise to INs, it is not clear to us which systems are obtained when lifting the functional systems below to communicational ones. 


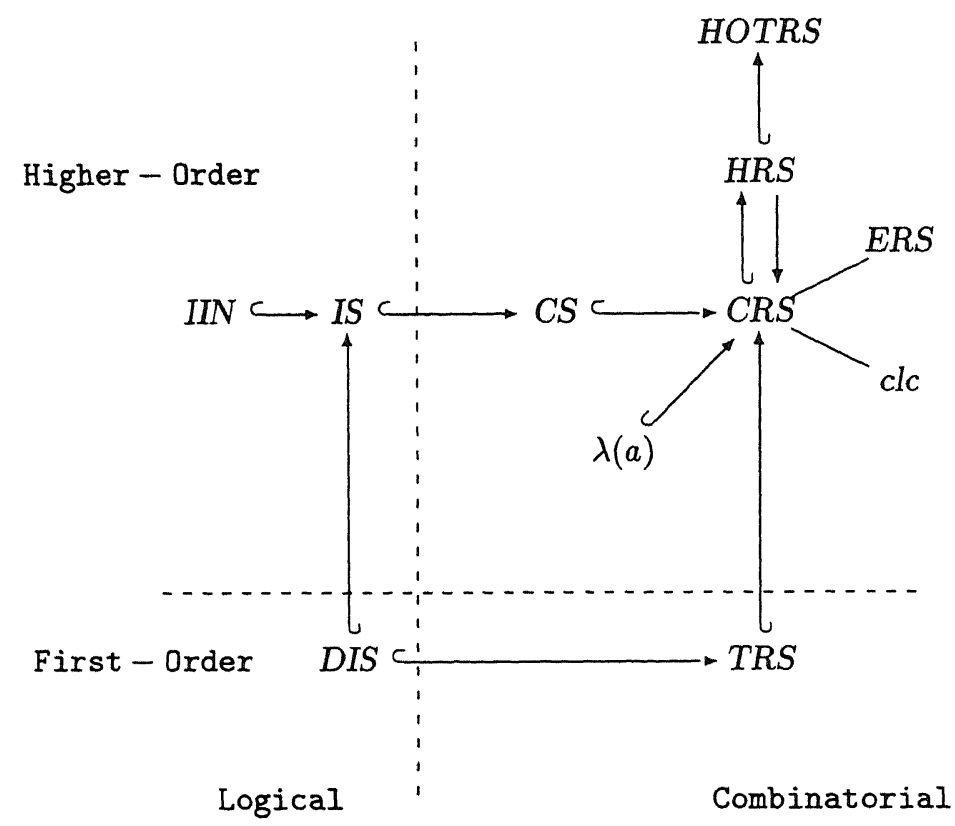

We will not discuss these systems here, but give references to the literature instead. For an overview of systems until 1980 see also [Klo80, pp. 132,133]. In (as far as we know) historical order we have:

- TRS = Term Rewriting System. We don't know who introduced this name, but they were known at the end of the seventies. Cf. also Rosen [Ros73].

- $C S=$ Contraction Scheme. Introduced by Aczel [Acz78].

- $\lambda(\mathrm{a})$-reductions were introduced by Hindley [Hin78].

- $C R S=$ Combinatory Reduction System. Introduced by Klop [Klo80].

- HOTRS $=$ Higher-Order Term Rewriting System. Introduced by Wolfram in his $\mathrm{PhD}$ thesis, see [Wol93].

- ERS = Expression Reduction System. Introduced by Khasidashvili [Kha90].

- (I)IN = (Intuitionistic) Interaction Net. Introduced by Lafont [Laf90].

- HRS = Higher-order Rewrite System. They were introduced by Nipkow [Nipa].

- $(D) I S=$ (Discrete) Interaction System. Introduced by Asperti and Laneve [AC92].

- $c l c=$ conditional $\lambda$-calculi. Introduced by Takahashi [Tak].

In general, if one system is encoded into another, it makes sense to check which syntactic properties are preserved by the translation. We will briefly review whether the (syntactic) properties (local) confluence and (weak) termination are preserved by the two encodings that are presented in this paper.

First we will consider the translation from CRSs into HRSs, say a CRS $\mathcal{C}$ is encoded into a HRS $\langle\mathcal{C}\rangle$. Since term formation in HRSs is quite liberal we will first restrict attention to the sub-HRS $\langle\mathcal{C}\rangle_{t r}$ with only the terms that are a 
translation of a term in $\mathcal{C}$. This is the HRS which we usually call 'the translation' of $\mathcal{C}$.

It is easily shown that if $\mathcal{C}$ is (locally) confluent, then its translation $\langle\mathcal{C}\rangle_{t r}$ is (locally) confluent. This is a consequence of Theorem 3.11 and 3.8. It is also easily shown that $\langle\mathcal{C}\rangle_{t r}$ is (weakly) terminating if $\mathcal{C}$ is (weakly) terminating. Note that we have as a corollary of Theorem 3.11 that the translation of a normal form is again a normal form.

If we, just out of curiousity, consider not the translation $\langle\mathcal{C}\rangle_{t r}$ of a CRS $\mathcal{C}$ but the full HRS $\langle\mathcal{C}\rangle$, then the situation is completely different. This is due to the fact that the rewrite relation for CRS is defined only on the set of terms (not containing metavariables), whereas in a HRS there is no syntactic difference between metavariables and variables.

Confluence nor local confluence are preserved if a CRS $\mathcal{C}$ is encoded into a HRS $\langle\mathcal{C}\rangle$. Consider the $\operatorname{CRS} \mathcal{C}_{1}$ :

$$
\begin{aligned}
F(F(Z)) & \rightarrow F(Z) \\
F([x] Z(x)) & \rightarrow F([x] Z(Z(x))) \\
F([x] Z(x)) & \rightarrow F(Z(A))
\end{aligned}
$$

It is confluent. This is a consequence of the fact that we have only one operator symbol $F$, for which we have the first rule. The corresponding HRS $\left\langle\mathcal{C}_{1}\right\rangle$

$$
\begin{aligned}
F(F z) & \rightarrow F z \\
F(\Lambda \lambda x . z x) & \rightarrow F(\Lambda \lambda x . z(z x)) \\
F(\Lambda \lambda x . z x) & \rightarrow F(z A)
\end{aligned}
$$

is not confluent: the term $F(\lambda x . z x)$ can be rewritten to $F(z A)$ by applying the last rule, and to $F(z(z A))$ by applying the second and then the third rule. Note that $F(\lambda x . z x)$ is not the translation of a term in $\mathcal{C}_{1}$. Note that $\left\langle\mathcal{C}_{1}\right\rangle$ is not locally confluent either.

The same holds for the properties weak termination and termination: they are not preserved by the translation from CRSs into HRSs. Consider the CRS $\mathcal{C}_{2}$ :

$$
F([x][y] Z(x, y)) \rightarrow Z([x][y] Z(y, x),[x] x)
$$

It is terminating and thus weakly terminating. This can be understood by remarking that the alphabet contains only one operator symbol, which is unary. So an instance of $Z(x, y)$ will contain exactly one variable, and never both $x$ and $y$. The corresponding $\operatorname{HRS}\left\langle\mathcal{C}_{2}\right\rangle$

$$
F(\Lambda \lambda y . z x y) \rightarrow z(\Lambda \lambda x \Lambda \lambda y . z(\Lambda \lambda x \Lambda \lambda y . z y x)(\Lambda \lambda x . x)
$$

is not terminating: the term $F(\Lambda \lambda x . \Lambda \lambda y . z(F x)(F y))$ permits an infinite rewrite sequence. Note moreover that it hasn't got a normal form, so it is not weakly terminating either. If we would consider the properties for rewriting on metaterms, then we conjecture that the properties are all preserved. 
Now we will consider the translation from HRSs into CRSs, say $\mathcal{H}$ is translated into $\langle\mathcal{H}\rangle$. Again we consider first the sub-CRS $\langle\mathcal{H}\rangle_{t r}$ of $\langle\mathcal{H}\rangle$ where we only consider the terms that are a translation of a term in $\mathcal{H}$. This is the CRS which we usually call 'the translation'.

As a consequence of Lemma 4.21 and Theorem 4.10, we obtain that $\langle\mathcal{H}\rangle_{t r}$ is confluent if $\mathcal{H}$ is confluent. It is also easily shown that weak termination and termination are preserved.

Again, the situation is completely different if we consider the full CRS $\langle\mathcal{H}\rangle$. In this case, this is due to the fact that the untyped $\beta$-rule is always present in the CRS which is associated to a HRS.

The HRS $\mathcal{H}_{1}$

$$
D x x \rightarrow E
$$

is confluent, but the associated CRS $\left\langle\mathcal{H}_{1}\right\rangle$

$$
\begin{aligned}
D(Z, Z) & \rightarrow E \\
@\left([x] Z(x), Z^{\prime}\right) & \rightarrow Z\left(Z^{\prime}\right)
\end{aligned}
$$

is not confluent. This has been proven by Klop (see [Klo80]).

For an arbitrary HRS $\mathcal{H}_{2}$, its associated CRS $\left\langle\mathcal{H}_{2}\right\rangle$ is terminating nor weakly terminating. This is the case since $\left\langle\mathcal{H}_{2}\right\rangle$ will contain for instance the term $@([x] @(x, x),[x] @(x, x))$. So in particular the CRS associated to a HRS that is terminating is not terminating, and the same holds for weak termination.

In this paper we have shown two extensions of first-order rewriting to higherorder, CRSs and HRSs, to be almost equivalent. The difference lies in the metalanguage used; they employ different flavours of the $\lambda$-calculus to generate their rewrite relations. For CRSs the underlined $\lambda$-calculus is used, while for HRSs the simply typed $\lambda$-calculus is used.

The translations from one system to the other are relatively simple because both are based on $\lambda$-calculus. The situation would be different for arbitrary metalanguages. But in fact it is hard to imagine a meta-language essentially different from $\lambda$-calculus. The basic steps of a rewrite (or redex-reaction) are: decomposing an object into a context and a redex, decomposing a redex into a pattern and a substitution, replacing the pattern with some other pattern, and then composing everything in the reverse order. The $\lambda$-calculus can be viewed as a 'calculus of (de)composing', so seems to be basic to any meta-language. If we look at other higher order rewrite formalisms, such as the Expression Reduction Systems of Khasidashvili [Kha90] and the Conditional Lambda Calculi of Takahashi [Tak], this claim seems to be supported. The precise interrelation is left to future work. We do note however that the similarities between these systems are obfuscated by the surprisingly large syntactical differences.

The work in this paper seems to suggest that only two basic properties are required for the flavour of $\lambda$-calculus one uses for the meta-language: confluence and termination. One can view CRSs and HRSs then as special cases of such a unifying theory of Higher Order Rewriting Systems (HORS). A large part of the syntactic rewrite theory should carry over to higher-order rewriting with more 
powerful meta-languages such as higher-order $\lambda$-calculi. Progress in this respect has been made in [Oos94b] and [OR94].

\section{Acknowledgements}

We would like to thank Fer-Jan de Vries for comments on an earlier version of this paper. We have benefitted from discussions with and between Jan Willem Klop, Tobias Nipkow and Stefan Kahrs on this subject.

\section{References}

[AC92] A. Asperti and Laneve C. Interaction systems I: the theory of optimal reductions. Technical Report 1748, INRIA-Rocquencourt, September 1992.

[ACCL90] M. Abadi, L. Cardelli, P.-L. Curien, and J.-J. Lévy. Explicit substitutions. In Proceedings of the ACM Conference on Principles of Programming Languages, San Francisco, 1990.

[Acz78] Peter Aczel. A general Church-Rosser theorem. Technical report, University of Manchester, July 1978.

[Bar84] H.P. Barendregt. The Lambda Calculus, its Syntax and Semantics, volume 103 of Studies in Logic and the Foundations of Mathematics. North-Holland Publishing Company, revised edition, 1984. (Second printing 1985).

[BG93] M. Bezem and J.F. Groote, editors. Proceedings of the International Conference on Typed Lambda Calculi and Applications, volume 664 of Lecture Notes in Computer Science, Utrecht, The Netherlands, March 1993. Springer-Verlag.

[BTG89] V. Breazu-Tannen and J. Gallier. Polymorphic rewriting conserves algebraic strong normalization and confluence. In Proceedings of the 16th International Colloquium on Automata, Languages and Programming, volume 372 of Lecture Notes in Computer Science, pages 137-150, 1989.

[Hin78] R. Hindley. Reductions of residuals are finite. Transactions of the American Mathematical Society, 240:345-361, June 1978.

[Kah92] S. Kahrs. Context rewriting. In M. Rusinowitch and J.L. Rémy, editors, Proceedings of the Third International Workshop on Conditional and Typed Rewriting Systems, pages 21-35, 1992.

[Kha90] Z.O. Khasidashvili. Expression reduction systems. In Proceedings of $I$. Vekua Institute of Applied Mathematics, volume 36, pages 200-220, Tbilisi, 1990.

[Klo80] J.W. Klop. Combinatory Reduction Systems. Mathematical Centre Tracts Nr. 127. CWI, Amsterdam, 1980. PhD Thesis.

[KOR93] J. W. Klop, V. van Oostrom, and F. van Raamsdonk. Combinatory reduction systems, introduction and survey. Theoretical Computer Science, 121(1-2):279-308, December 1993.

[Laf90] Yves Lafont. Interaction nets. In Proceedings $17^{\text {th }}$ ACM Symposium on Principles of Programming Languages, pages 95-108, 1990.

[Lan93] C. Laneve. Optimality and Concurrency in Interaction Systems. PhD thesis, dipartimento di informatica università di pisa, March 1993. 
[LIC91] Amsterdam, The Netherlands. Proceedings of the sixth annual IEEE Symposium on Logic in Computer Science, Los Alamitos, July 1991. IEEE Computer Society Press.

[Mil] D. Miller. A logic programming language with lambda-abstraction, function variables, and simple unification. In [Sie91].

[Nipa] T. Nipkow. Higher-order critical pairs. In [LIC91].

[Nipb] T. Nipkow. Orthogonal Higher-Order Rewrite Systems are Confluent. In [BG93].

[NM88] G. Nadathur and D. Miller. An overview of $\lambda$ Prolog. In R.A. Kowalski and K.A. Bowen, editors, Proc. 5th Int. Logic Programming Conference, pages 810-827. MIT Press, 1988.

[Oos94a] V. van Oostrom. Confluence by decreasing diagrams. Theoretical Computer Science, 1994.

[Oos94b] V. van Oostrom. Confluence for Abstract and Higher-Order Rewriting. $\mathrm{PhD}$ thesis, Vrije Universiteit, March 1994.

[OR93] V. van Oostrom and F. van Raamsdonk. Comparing combinatory reduction systems and higher-order rewrite systems. Technical Report CS-R9361, CWI, September 1993. also available as VU technical Report IR-333.

[OR94] V. van Oostrom and F. van Raamsdonk. Weak orthogonality implies confluence: the higher-order case. In Proceedings of the Symposium on Logical Foundations of Computer Science 1994, 1994. to appear.

[Pau90] L.C. Paulson. Isabelle: The next 700 theorem provers. In P. Odifreddi, editor, Logic and Computer Science, pages 361-385. Academic Press, 1990.

[Raa93] F. van Raamsdonk. Confluence and superdevelopments. In C. Kirchner, editor, Proceedings of the 5th International Conference on Rewrite Techniques and Applications, 1993.

[Ros73] Barry K. Rosen. Tree-manipulating systems and Church-Rosser theorems. Journal of the Association for Computing Machinery, 20(1):160-187, January 1973.

[Sie91] J. Siekmann, editor. Extensions of Logic Programming, volume 475 of Lecture Notes in Artificial Intelligence. Tübingen, FRG, Springer-Verlag, December 1991.

[Tak] M. Takahashi. $\lambda$-calculi with conditional rules. In [BG93].

[Wol93] D.A. Wolfram. The Clausal Theory of Types, volume 21 of Cambridge Tracts in Theoretical Computer Science. Cambridge University Press, 1993. 